Bull, Mater. Sci., Vol. 4., No. 2, April 1982, pp. 167-174. (C) Printed in India.

\title{
Electrochemical experiments in space
}

\author{
S R RAJAGOPALAN \\ Materials Science Division, National Aeronautical Laboratory, Bangalore 560017 \\ India
}

MS received 29 March 1982

\begin{abstract}
The probable effects of rarefied atmosphere and near-zero gravity conditions that prevail in space, in relation to electrochemical experiments, are briefiy discussed. The various space electrophoresis experiments are reviewed and discussed. Some possible additional electrochemical experiments are suggested.
\end{abstract}

\section{Intraduction}

Space environment is characterised by the absence of atmosphere and a near-zero gravity condition (the so-called micro-gravity). In the case of molten salt electrochemical systems, the lack of atmosphere could be an advantage since the molten salt and the products of electrolysis will not have any interaction with atmosphere. Aqueous and non-aqueous electrochemical systems will pose problems in such rarefied atmospheres due to the voltalisation of high vapour pressure solvent which will demand closed circuit. Apart from these experimental problems, no significant effect is likely to arise from the lack of atmosphere in space.

The near zero gravity condition can have significant effect on electrochemical experiments. By and large electrochemical systems are governed by Nernst equation, Faraday's laws of electrolysis, and rate law. The constants occurring in these laws are not influenced by $G$. The rate is however dependent upon mass transfer which can under certain circumstances be influenced by $G$.

The most important effects of near-zero gravity from the point of view of an electrochemist is as follows :

(i) almost zero convection current due to density gradient.

(ii) absence of sedimentation of particles.

These effects lead to a better electrophoretic separation in space as compared to $1 \mathrm{G}$ condition. We shall briefly review here the electrophoresis experiments in space. We shall also attempt at suggesting certain other interesting electrochemical experiments that can be done in space and briefly dwell on the utility of such experiments. 


\section{Electrophoresis}

In biological research there is always a necessity of separating a single type of cell from a mixture and also isolating specific macromolecules from biological fluids which contain several types of them. Several of the separation technique is not well suited for the task of cell separation since they damage them (Ramaseshan 1978). Electrophoresis is a well-established electrochemical separation technique for the type of materials referred to above.

Electrophoresis is the electrokinetic method that physically separates charged particles in an aqueous medium under the influence of an electric field. Many different types of electrophoresis have been developed since Picton and Linder introduced the moving boundary technique which was made into an acceptable practical method for separation by Tiselius by developing a substantially improved version of the apparatus (Picton and Linder 1892 ; Tiselius 1937). This subject has been reviewed extensively (Moore 1960 ; Tiselius 1968).

In moving boundary electrophoresis, the sample is introduced into a stationary medium (buffer) in which two electrodes are placed. On applying a field, the charged species migrate to the oppositely charged electrode at different rates (governed by charge and mass of particle) and separate into zones. This technique is frequently referred, in space literature as free zone electrophoresis.

Isotachophoresis and isoelectric focussing can be regarded as interesting modifications of free zone electrophoresis and are briefly described below.

Isotachophoresis (from the Greek meaning 'equal speed ') consists of placing a mixture of charged particles (sample) of the same sign (co-ions) in a column, containing in addition two electrolytes, one of which has co-ions more mobile than any sample co-ions, while the other electrolyte has co-ions less mobile than any sample co-ion, the entire electrolyte having a common counter-ion. Upon applying a constant d.c. current, the sample co-ions initially move at different velocities until a steady state is reached in which the sample ions separate into continuous zones with sharp interfaces in order of their mobilities. The zones, thereafter, migrate at the same velocities and adjust themselves into compartments of various lengths, concentrations and conductivities such that the product of the co-ion mobility and the field gradient is the same within each zone. The polarity of the electric field must be such that the leading ion migrates towards the electrode that is placed on the same side of the sample as the leading electrolyte.

In isoelectric focussing the charged particles of the sample is made to migrate under an electrical field in an electrolyte medium having a $\mathrm{pH}$ gradient. The charged particle (of sample) becomes neutral when it reaches the $\mathrm{pH}$ that represents its isoelectric point and becomes stationary. Thus the sample is separated into species of equal isoelectric point.

The different types of electrophoresis described above are useful for the qualitative analysis but are of marginal value as preparative techniques since their through-put is very poor. Flow electrophoresis or continuous flow electrophoresis is well suited for preparative purposes. In this method the buffer solution is made to flow. The sample is continuously fed into the flowing liquids (figure 1a). An electric field is applied perpendicular to the flow direction. The sample undergoes 

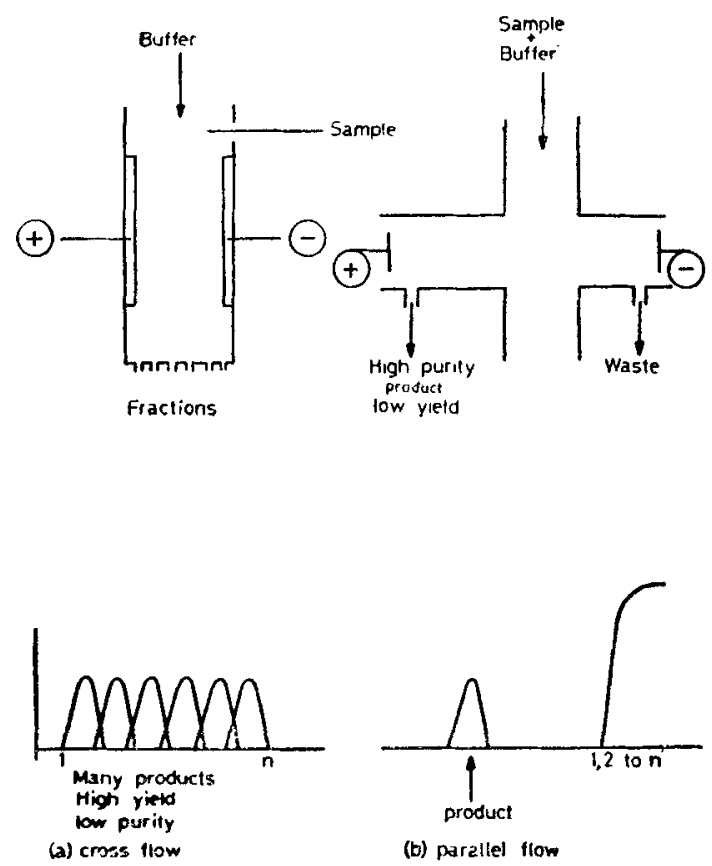

Figure 1. Modes of contiunous flow electorphoresis.

separation during the residence time of the sample. By employing tapping ports, many products can be collected at high yield and low purity. If the electric field is applied parallel to the flow as in figure $1 \mathrm{~b}$ then one of the products can be recovered at high purity, the yield however will be low.

\section{Free zone electrophoresis and isotachophoresis experiments in space}

Electrophoresis in a free fluid was first demonstrated in space during the Apollo 14 mission and was followed in the Apollo 16 mission (Snyder et al 1973) and in ASTP experiment MA-011. The Apollo 16 electrophoresis demonstration was performed using two different sizes of polystyrene latex particles. The results failed to yield much more than the proof that electrophoretic separation under micro-gravity can be achieved without sedimentation of particles.

The ASTP experiment MA-011 aimed at the electrophoretic separation of human lymphocytes, aldehyde fixed red blood cells and human kidney cells. Equipment problems during the attempted runs of lymphocytes prevented the acquisition of data that could have proved whether it is possible to obtain pure fractions of lymphocytes from heterogeneous cell populations.

Rabbit, human and horse red cells were partially resolved. The mean mobilities at the nearest neighbour populations (in this case) were separated by about $0.4 \mu \mathrm{m} \mathrm{S}^{-1} \mathrm{~V}^{-1}$. Therefore successful separation is not trivial. 
Human foetal kidney cells produce the enzyme urokinase (UK) which is able to accomplish blood clot lysis and hence the interest in its commercial production for therapeutic purposes. Unfortunately only $5 \%$ cells in the cortex of the kidney produce UK (Bermic and Kwaan 1969). If these UK producing cells could be isolated and subcultured a 20 fold increase in production could probably be achieved. The kidney cell separation in ASTP experiment MA-011 was most encouraging. One of the separated band of cells on reculturing produced predominantly UK. The other band of cells on reculturing gave human granulocyte stimulating hormone as the prime product. This finding shows that the UK producing cells could be successfully separated in space, returned and cultured, thereby showing the possibility of using space electrophoresis for the commercial production of UK.

The isotachophoresis conducted in ASTP experiment MA-011, though demonstrated the remarkable sharpness of isotachophoretic boundaries was of limited value since equipment problems limited the value of the results. The potential application of this method in microgravity environment has been reviewed by Bier et al (1975). But the potentialities still remain unexplored.

\section{Continuaus flow electropharesis experiments in space}

The configuration for continuous flow electrophoresis is a rectangular parallelopiped with height, $h$, large relative to the width, $w$, and the depth $d$. The ratio $d / w$ is also small. The electrode of length $l$ is located on the end walls such that $w$ represents the interelectrode distance. In this set-up the product form bands, parallel to the long axis. The occurrence of natural convection is detrimental because it results in a mixing of the fluid.

Two modes of natural convection can be distinguished : (i) conventional convection, (ii) unstable convection. These can either occur separately or together in a given configuration. Conventional convection is generated by a density gradient that is normal to the gravitational vector. This therefore results when the wall temperature and fluid temperature are different due to joule heating and wall cooling. Unstable convection can occur when the density gradient is parallel to but opposed to gravity vector. In this case, unlike conventional convection, the onset of motion is not immediate but starts when a critical temperature (density) gradient is exceeded. Once it starts it causes a lot more of mixing. The temperature gradient for this case is vertical, i.e., the one that occurs when the fluid is passed down. Ostrach has presented a detailed analyses of both the types of convection (Ostrach 1976). These convectional disturbances are minimal if the interelectrode distance (gap) is small. But that affects the through-put. Thus through-put and resolution are opposed to each other under $1 \mathrm{G}$ condition. However, under micro. gravity condition, greater through-put can be achieved without sacrificing resolution by increasing the gap, since convection is virtually absent. Hence space environment offers specific advantage for preparative electrophoresis.

Continuous flow electrophoresis was attempted in ASTP experiment MA-014. A lot of experimental difficulties like long sample rest periods, absolute sealing of the fluid loops, resuspension of the sample in the absence of gravity and sepa- 
ration and absorption of the hydrogen produced during electrophoresis were met with and were solved. The unsatisfactory working of the optical system imposed a limitation on the extent of availability of result. Rat bone marrow, rat spleen cells, rabbit and human erythrocytes and lymph node of rat were subjected to electrophoresis. The results in spite of the malfunctioning of the optical system show high resolution capability. The through-put was $7 \mathrm{~cm}^{3} / \mathrm{hr}$ whereas comparable ground equipment would have a through-put of $1.2 \mathrm{~cm}^{3} / \mathrm{hr}$.

\section{Discussion on the space electrophoresis experiments}

Reviewing the results of batch type electrophoresis and continuous flow electrophoresis carried out in space, one finds that the runs yielded only partial qualitative results due to sample equipment and retrieval failures. It is probable that more significant results could have been obtained had a more fruitful and less ambitious course been followed in the planning of the experiments ; no two runs were performed to separate the same initial mixture; no two flights carried exactly the same equipment. In spite of all these limitations the space electrophoresis experi ments have established (1) good resolution could be achieved; (2) higher throughput could be obtained. With the exception of kidney cell separation, the space electrophoresis experiments have not shown it to be phenomenally different from that performed under $1 G$. This merits a detailed examination. Perhaps it is worthwhile to try out the suggestions of Ostrach to improve the through-put of $1 \mathrm{G}$ continuous flow electrolysis and compare it with space data.

\section{Other electrochemical experiments that might be possible}

\subsection{Preparation of electrocomposites}

Electroplating from plating baths containing suspended solids results in incorporation of the suspended solid (conducting or non-conducting) into the deposit. For example if $\mathrm{Al}_{2} \mathrm{O}_{3}$ were to be suspended in nickel plating bath and plated then the nickel plate contains a dispersion of $\mathrm{Al}_{2} \mathrm{O}_{3}$. In other words the suspension electrolysis has resulted in a composite of $\mathrm{Ni}+\mathrm{Al}_{2} \mathrm{O}_{3}$. Since this composite has been produced by electrolysis, it is referred to as electrocomposite. Such composites can be tailored for improved wear resistance, abrasion resistance, for self lubrication or for cutting tool application.

The mechanism of 'Code position' of the suspended solid is little understood. Several theories like electrophoretic deposition, adsorption, etc., have been proposed. The experiments (Rajagopalan and Indira Rajagopalan) done by us in NAL makes us postulate the following hypothesis:

The suspended particles $\left(\mathrm{Al}_{2} \mathrm{O}_{3}, \mathrm{SIC}, \mathrm{WC}\right.$, etc.) adsorb the metal ions say nickel ions. The particles are kept suspended by agitation. The particles of suspended solid moving within the liquid can hit the cathode. Some of them will bounce. A few can come to rest at the cathode surface because their kinetic energy is just equal to the energy absorbed by the metal on impact. Such particles would 
begin to fall down because of gravity. Due to roughness on the metal surface and that on the particle, the particle can remain in contact with the cathode for a short time. In this time, the ions adsorbed on to the particle near the particle cathode interface may get reduced thereby bonding the particle to the metal surface. Thereafter the particle gets progressively coated and is finally embedded.

Since in space environment, sedimentation is absent, it should be quite easy to keep the particles suspended in the plating bath even when the percentage of suspended solid is high. Particles, coming to rest on hitting the cathode, will remain on the cathode surface and will not fall down (due to near-zero gravity condition). If the mechanism postulated above is correct, then the ' Code position 'of particle will be more. One might expect that in space environment electrocomposites with higher volume fraction of dispersed phase and those with coarser particles should be easily prepared.

\subsection{Preparation of metal powder by electrolysis}

When metal salt solutions are electrolysed at current densities approaching the limiting current density (i.e. the current density that can be supported by the maximum rate of supply of the ion getting reduced or the current density at which the surface concentration of the metal ion is virtually zero at the cathode surface) the deposit is in the form of powder. The condition leading to powder formation can create conditions very favourable for convection. As a result of convection the limiting current density will be increased and the limiting current density may not be uniform on the cathode covered with a powder. Therefore the particle size of the powder may vary from point to point on the cathode and may vary with time due to the convection current. In space environment, since convection is absent one might expect more uniform powder.

\subsection{Deposition of smoothed coatings}

Thick layers of electroplates tend to be rough perhaps due to varying amounts of mass transport which in turn stems from the variations in the convection resulting from density gradients. Absence of convection in space should therefore yield smoother deposit.

\subsection{Detachment of gas bubbles from electrode surface}

Padday (1976) showed that the pressure inside a bubble sticking to the surface is given by

$$
P=r_{L}\left(\frac{1}{R_{V}}+\frac{1}{R_{L}}\right)+\rho g h
$$

where $P$ is the pressure inside the bubble; $r_{L}$ is the surface free energy of liquid/ vapour interface ; $R_{V}$ and $R_{L}$ are the two principal radii of curvature of the bubble; $\rho g h$ is the hydrostatic pressure due to a liquid column of $h \mathrm{~cm}$ above the bubble. 


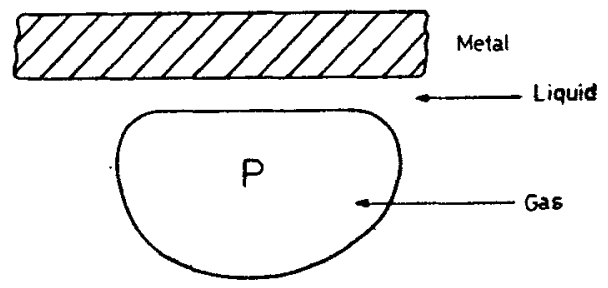

(a) Stable

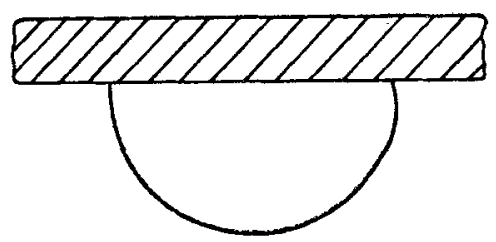

(b) Unstable

Figure 2. Attachment of gas bubble.

This pressure acts on the thin film of liquid between the bubble and the metal (figure 2) and is trying to push it away from the surface. It is the force of attraction between the solid and the liquid that is preventing the liquid draining away under this pressure which has been named the 'disjoining pressure' by Derjaguin. Under $1 \mathrm{G}$ conditions the larger value of disjoining pressure leads to the unstable attachment of bubble (figure $2 b$ ). Under microgravity condition the stable attachment (figure $2 a$ ) results, since $\rho g h$ tends to zero.

Applying the ideas developed above to the formation of gas bubble, formation due to electrolysis (say hydrogen evolution) the following conclusions may be reached. Under terrestrial or $1 G$ conditions the bubble will have an unstable attachment while under space condition it will be stably attached. The absence of buoyancy will therefore make it difficult to dislodge it. Hence in space electrolysis, gas bubble sticking may pose problem by shielding the area under the bubble and altering the current distribution.

\section{References}

Bermic M B and Kwaan H C 1969 Plasninogen activator activity in cultures from human tissues. An immunological and histachemical study. J. Chem. Invest. 481740

Bier M, Hinckley J O N and Smolka A J K 1975 Potential use of isotachophoresis in space protides of the biological fluids, 22nd colloquium (ed.) H Peeters (New York : Pergamon Press) pp. 673-678

Moore D H 1960 Electrophoresis in physical methods or organic chemistry (Technique of Organic Chemistry, Vol. 1) (ed.) A Weissberger 3rd ed. Part IV (New York : Interscience) p. 3113 
Ostrach S 1976 The influence of convection in continuous flow electrophoresis, ESA Special Publication No, 114, 141-147

Padday J E 1976 Capillary forces and stability in zero-gravity environments, ESA Special Publication No. 164, 447-454

Picton H and Linder S L 1892 J. Chem. Soc. 61148

Rajagopalan S $\mathbf{R}$ and Indira Rajagapalan, Unpublished results

Ramaseshan S 1978 Processing of materials in space, Scientific note ISRO-SN_B4-78

Seasmen G V F, Allen R E, Barlow G H and Bier M 1976 Detailed results of ASTP experi ments, MA-011-ESA Publication No. 114, 155-166

Snyder R S, Bier M, Griffin R N, Johnson A L, Leidheiser H, Micale F J, Van der hoff J W Ross S and Van Oss C J 1973 Free fluid particle electrophoresis on Apollo 16, Separ. Purif. Methods 2 259-282

Tiselius A 1937 Trans. Faraday Soc. 33524

Tiselius A 1968 Ann. Rev. Biochem. 37 